\title{
OPTICAL DATA STORAGE USING A SOLID IMMERSION LENS (Invited)
}

\author{
H. J. MAMIN, B. D. TERRIS and D. RUGAR \\ IBM Research Division, Almaden Research Center, 650 Harry Road, San Jose, California 95120-6099, USA
}

\begin{abstract}
A near-field optical technique, using a new type of Solid Immersion Lens (SIL), has been developed and applied to the writing and reading of domains in a magneto-optic material. The SIL is a truncated glass sphere which serves to increase the numerical aperture of the optical system by $n^{2}$, where $n$ is the index of refraction of the lens material. By mounting a $1 \mathrm{~mm} \mathrm{SIL} \mathrm{on} \mathrm{a} \mathrm{slider,} \mathrm{we} \mathrm{have} \mathrm{been} \mathrm{able} \mathrm{to} \mathrm{fly} \mathrm{the} \mathrm{SIL} \mathrm{on} \mathrm{a}$ magneto-optical disk. We have demonstrated writing and reading with such a lens at data rates exceeding 1 Mbit/s with a carrier-to-noise ratio of approximately $50 \mathrm{~dB}$. Using a $830 \mathrm{~nm}$ laser and a $n=1.5 \mathrm{SIL}$, the focused spot size was reduced by close to a factor of 2 and was measured to be $600 \mathrm{~nm}$.
\end{abstract}

KEYWORDS: NEAR-FIELD OPTICS, OPTICAL RECORDING, HIGH DENSITY STORAGE

To increase the data density in optical recording, it is desirable to reduce the optical spot size. One approach to obtaining a smaller spot size is to use a pin hole aperture to circumvent the diffraction limit. Recently, near-field optical techniques have been developed to accomplish this [1-3]. In particular, Betzig et al.[4] have used a sub-micron aperture operating in the near-field regime to write and image magneto-optical (MO) bits at high density. Alternatively, the diffraction-limited spot size can be reduced by decreasing the wavelength or by increasing the numerical aperture (NA). By decreasing the wavelength, a density of $2.4 \mathrm{Gbits} / \mathrm{sq}$. in. has been achieved using blue light $[5,6]$. Further advances, however, will likely require innovation in increasing the NA.

Kino and co-workers $[7,8]$ have demonstrated an approach to increasing the NA above the theoretical upper limit of 1 which is obtainable in air by using an immersion lens made from a high index of refraction $(n)$ material, the Solid Immersion Lens (SIL). The lens is formed by placing a truncated sphere between a focusing objective and the sample, with the flat side facing the sample (Fig. 1). The wavelength inside the sphere is reduced by the high index of the glass, leading to a reduction in the diffraction limited spot size. In addition, if the incident rays are refracted at the sphere's surface, the maximum incident angle in the lens is increased above that in air, leading to a further reduction in spot size. The large angle rays, however, may be at angles greater than the critical angle for exiting the high index glass at the base of the SIL.
Thus, both the reduced wavelength and large angle rays exist only within the high index SIL and must be coupled via their evanescent field to the sample located at the base of the SIL. By placing the substrate within the evanescent decay length, the small spot can be transmitted across the air gap. This is, therefore, a form of near-field optics.

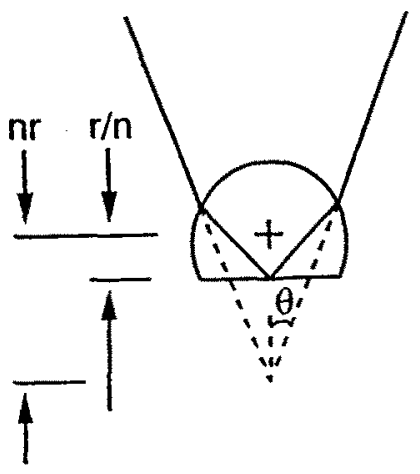

Figure 1. Schematic of the SIL concept. $n$ is the index of refraction of the sphere and $r$ its radius.

There are two special truncated spheres for which converging incident rays are focused without aberration at the base of the partial sphere. One truncated sphere for which such aberration free focusing occurs is the hemisphere. In this case rays, focused to the center of the hemisphere's base, are incident normal to the spherical surface and are therefore not refracted. The wavelength, however, is reduced by a factor of $n$ and the effective NA is increased by $n$. Mansfield and Kino 
[7] demonstrated imaging with such a hemispherical glass lens and resolved a $200 \mathrm{~nm}$ period resist grating using $436 \mathrm{~nm}$ light.

There is a second type of partial sphere for which aberration free focusing occurs $[9,10]$. This occurs when the sphere is truncated to a thickness $r(1+1 / n)$, where $r$ is the radius of the sphere, as shown in figure 1. A focused spot will be obtained at the base of the SIL when the incident rays are converging toward a point located a distance $n r$ below the center of the sphere. Because the rays are refracted at the spherical surface, $\theta$ is increased. This increase in $\theta$, together with the reduction in $\lambda$, results is an increase in the NA by a factor of $n^{2}$ as compared to the incident NA. The maximum possible NA, however, cannot exceed $n$. By using a focusing objective with a NA $=0.55$, typical of those used in $\mathrm{MO}$ recording, and a solid immersion lens made of $n=1.9$ index glass, an effective NA of 1.9 can be obtained. The focused spot is thereby reduced by $3.5 \mathrm{x}$ and the potential bit density increased by an order of magnitude, without the use of shorter wavelength lasers. The SIL, can, of course, be combined with such short wavelength lasers, resulting in a theoretical spot size of approximately $125 \mathrm{~nm}$ and a bit density approaching $40 \mathrm{Gbits} / \mathrm{sq}$. in.

Previously, by placing this second type of SIL in contact with MO media, we have demonstrated writing and reading of $350 \mathrm{~nm}$ bits at low data rates [11]. In this work, we present results on a SIL flying above a spinning disk. Except for the addition of the SIL placed between the focusing objective and the MO media, we have used a standard optical recording setup, including differential detection of the Kerr rotation, as shown in figure 2. An $830 \mathrm{~nm}$ laser diode was used, along with a $\mathrm{NA}=0.5$ objective lens. The MO media was $\mathrm{Si}_{3} \mathrm{~N}_{4} / \mathrm{TbFeCo} / \mathrm{Si}_{3} \mathrm{~N}_{4}$ sputtered onto a glass disk. The laser light was incident from the media side of the disk. The SIL was a $n=1.5,1 \mathrm{~mm}$ diameter, glass sphere which was polished and mounted onto a slider. The slider had an air bearing pattemed onto it in the same manner as used in magnetic recording, allowing it to fly. While a flying lens has previously been tested for optical recording [12], it was used for the purpose of reducing access time and was not made part of the air-bearing surface.

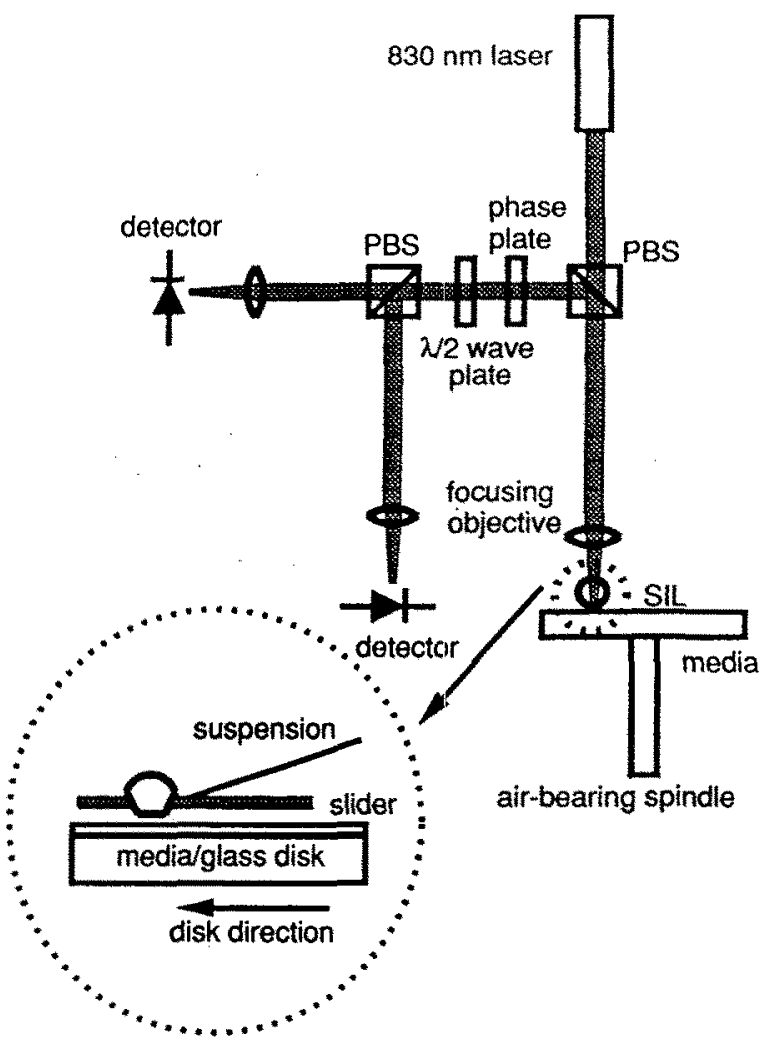

Figure 2. Schematic of the optical storage test setup with the SIL inserted between a focusing objective and optical disk. The SIL is mounted on a magnetic storage type slider to allow it to fly on the disk surface.

Figure 3 shows the read signal, with and without the SIL, from a track written without the SIL. The signal with the SIL clearly shows a more square shape and steeper transitions at the edges of the bit, indicative of the smaller spot size. The spot size can be estimated by measuring the roll-off in signal amplitude with increasing linear bit density, as shown in figure 4. By fitting this data, the spot diameter when using the SIL is found to be approximately $600 \mathrm{~nm}$. This is 0.54 of the diameter measured without the SIL. Using the $n=1.5$ SIL, the expected spot size due to the increased NA is $n^{-2}=(1.5)^{-2}=0.44$ of the spot size obtained without the SIL. Thus the performance is slightly worse than that predicted. 


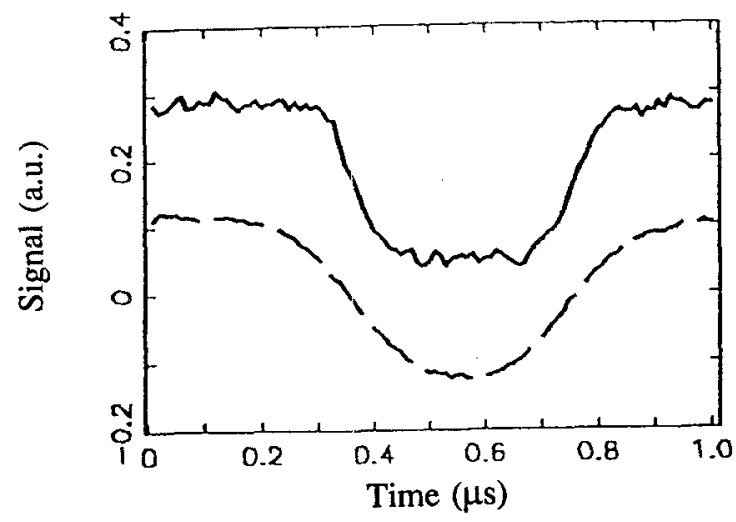

Figure 3. Waveform of a bit written without the SIL and read without (dashed) and with (solid) the SIL.

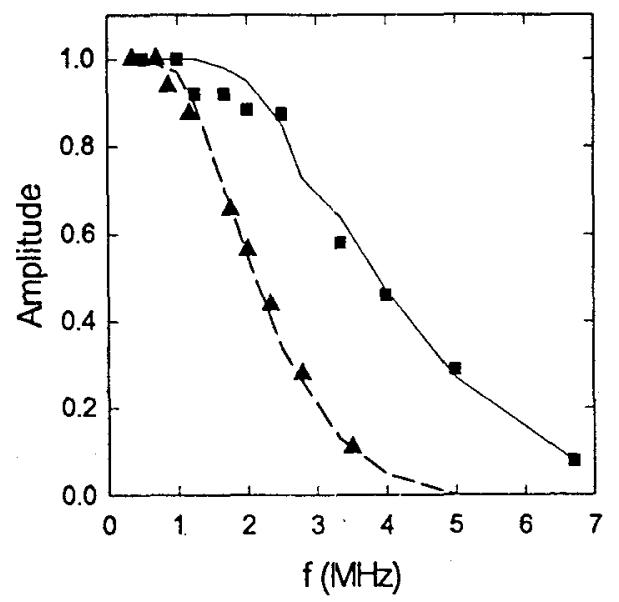

Figure 4. The signal amplitude as a function of data frequency with a $0.5 \mathrm{NA}$ focusing objective (triangles) and with the addition of the $n=1.5 \mathrm{SL}$ (squares). By fitting the roll-off curves, the focused spot size is estimated to be $1.1 \mu \mathrm{m}$ without the SIL and $0.6 \mu \mathrm{m}$ with the SIL.

Some of this less than theoretical performance is due to the slider fly height. We have measured the fly height of the slider as a function of disk velocity. The slider was seen to start flying at very low velocities and quickly reached a fly height of approximately $1.5 \mu \mathrm{m}$. It is at this fly height that the reading and writing experiments were performed. This height is much too high for the evanescent field from the SIL to couple to the disk, and therefore we would not expect the above critical angle rays radiating from the SIL to be able to propagate into the disk. Thus the maximum effective NA of this flying SIL should be limited to 1. This would limit the spot size to .5 of that achieved without the SIL, which is closer to the data of figure 4. This same conclusion is reached by calculating the spot size as a function of fly height using a program based on vector diffraction theory [13], where the spot size increases by roughly $10 \%$ when increasing the fly height from 0 to $2 \mu \mathrm{m}$.

To obtain a smaller focused spot size, it will be necessary to use a SIL made from higher index glass. The fly height will then become much more critical. Based on our calculations, if $n$ is increased to 1.8 , then even at a fly height of $0.1 \lambda$, the spot size is already increased by about $10 \%$. At a fly height of $0.5 \lambda$, the spot size has more than doubled and the transmitted intensity is dramatically reduced. Therefore, to realize such a high NA the SIL-media spacing will need to be well under a wavelength.

One of the challenges in implementing near-field data storage techniques is to do so at high data rates and with a reasonable carrier-to-noise ratio (CNR). Using the slider mounted SIL, it is possible to have a high relative velocity between the disk and the SIL. This, coupled with the high optical throughput of the SIL, makes achieving high data rates possible. As shown in figure 5, we have flown the SIL at a velocity of 4.7 $\mathrm{m} / \mathrm{s}$ and written and read back a $0.5 \mathrm{MHz}$ carrier. The apparent bit size is roughly 1 micron. The carrier-to-noise ratio (CNR) obtained when reading with the SIL is shown in figure 6 . In a $30 \mathrm{kHz}$ bandwidth we have achieved a CNR of nearly $50 \mathrm{~dB}$ at $1 \mathrm{MHz}$. This is about $10 \mathrm{~dB}$ less than that achieved without the SIL. However, the media has not been optimized for the small laser spot size. In order not to erase while reading using the SIL, the read power had to be reduced to $0.25 \mathrm{~mW}$, where the system was shot-noise limited. By optimizing the media, it should be possible to regain some of this lost CNR.

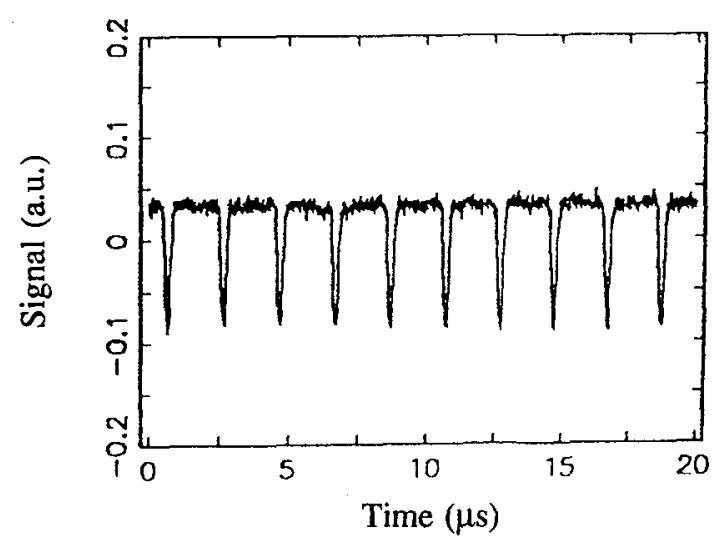

Figure 5. The readback signal using the SIL from a data track written with the SIL. 


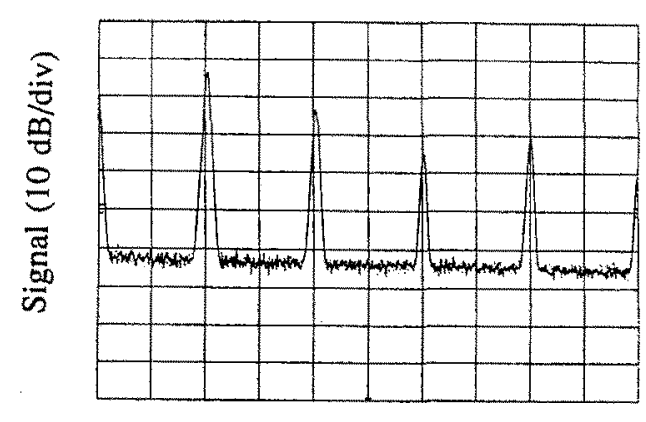

Frequency $(0.5 \mathrm{MHz} / \mathrm{div})$

Figure 6. CNR in a $30 \mathrm{kHz}$ bandwidth using the SIL.

We have demonstrated that a small spot size is achievable by flying a SIL above a MO disk surface. Considerable improvement in the performance of the SIL is possible by using a higher index of refraction glass. However, in order to realize the small focused spot size in such a SIL, it will be necessary to fly at a disk-SIL spacing of well under a wavelength. The SIL has excellent optical efficiency, which makes realistic data rates achievable at greatly enhanced densities. In addition, since the SIL operates in reflection, it is compatible the existing optical recording technologies. Further optimization should allow us to achieve blue light performance with a red laser. Using blue light, it should be possible to increase the storage density even further, to as high as 40 Gbits/in?2

1. D. W. Pohl, W. Denk, M. Lanz, Appl. Phys. Lett. 44, 651 (1984).

2. A. Lewis, M. Isaacson, A. Hartoonian, and A. Muray, Ultramicroscopy 13,227 (1984).

3. E. Betzig, M. Isaacson, and A. Lewis, Appl. Phys. Lett. 51, 2088 (1987);

4. E. Betzig, J. K. Trautman, R. Wolfe, E.M. Gyorgy, P.L. Finn, M.H. Kryder, and C.-H Chang, Appl. Phys. Lett. 61, 142 (1992).

5. J.E. Hurst, Jr. and W.J. Kozlovsky, Jpn. J. Appl. Phys. 32, 5301 (1993).

6. D. Rugar, C. J. Lin, and R. H. Geiss, IEEE Trans. Magn., MAG-23, 2263 (1987).

7. S. M. Mansfield and G. S. Kino, Appl. Phys. Lett. 57, 2615 (1990).

8. S. M. Mansfield, W. R. Studenmund, G. S. Kino, and K. Osato, Optics Lett. 18, 305 (1993).

9. M. P. Davidson, Proc. SPIE 1926, 84 (1993).

10. M. Bom and E. Wolf, "Principles of Optics," Pergamon Press, 1980, p.253.

11. B. D. Terris, H. J. Mamin, D. Rugar, W. R. Studenmund, and G. S. Kino, Appl. Phys. Lett, 65, 388 (1994).

12. K. Yasukawa, S. Yamaguchi, A. Murakami, H. Goto, N. Nishimura, and K. Ueyanagi, Jap. J. Appl. Phys. 28 (Suppl. 28-3), 205 (1989).

13. M. Mansuripur, J. Opt. Soc. Am. A 3, 2086 (1986) and J. Opt. Soc. Am. A 10, 383 (1993). 